\title{
Importance des infections à transmission vectorielle selon le système de production : trypanosomose bovine et dynamique d'innovation des éleveurs au Sénégal
}

\author{
F. Bouyer ${ }^{(1,2,3)^{*}}$, J. Bouyer ${ }^{(1,2,3)}$, M.T. Seck ${ }^{(1)}$, B. Sall ${ }^{(4)}$, A.H. Dicko ${ }^{(1)}$, \\ R. Lancelot ${ }^{(2,3)} \&$ E. Chia ${ }^{(5)}$ \\ (1) Institut sénégalais de recherches agricoles, Laboratoire national d'élevage et de recherches vétérinaires, \\ B.P. 2057, Dakar - Hann, Sénégal \\ (2) Unité mixte de recherche "Contrôle des maladies animales exotiques et émergentes ", Centre de \\ coopération internationale en recherche agronomique pour le développement (CIRAD), 34398, Montpellier, \\ France \\ (3) Unité mixte de recherche 1309 " Contrôle des maladies animales exotiques et émergentes ", Institut \\ national de la recherche agronomique (INRA), 34398, Montpellier, France \\ (4) Direction des Services vétérinaires, Dakar, Sénégal \\ (5) Unité mixte de recherche 0951 "Innovation », Institut national de la recherche agronomique - Centre de \\ coopération internationale en recherche agronomique pour le développement (INRA-CIRAD), 34398 Montpellier, \\ France \\ *Auteur chargé de la correspondance : neptisliberti@gmail.com, bouyer@cirad.fr
}

\section{Résumé}

Au Sénégal, un projet d'éradication d'une population de glossines (Glossina palpalis gambiensis) a été mis en place dans une région favorable à l'intensification de l'élevage, la région côtière des Niayes, dans le but de lever la contrainte trypanosomienne et de permettre une intensification écologique de la production bovine.

L'analyse croisée de dix études de cas constitue la phase inductive de l'évaluation de l'impact de la levée de cette contrainte sanitaire sur les trajectoires des stratégies d'élevage. Pour cela, la méthodologie de l'analyse compréhensive et des outils de l'épidémiologie participative ont été mobilisés afin de comprendre les logiques d'action. Les stratégies ont été analysées selon les objectifs des éleveurs et leurs capacités à mobiliser le réseau socio-technique, pour trois principaux types d'éleveurs : les agro-pasteurs, les agro-éleveurs et les intensifs laitiers.

Le risque trypanosomien constitue une contrainte intégrée dans les conduites d'élevage par le recours à la génétique trypanotolérante, la prophylaxie médicale ou le choix de zones à faible risque pour la localisation des élevages. Ainsi, la disparition du risque trypanosomien aura un impact majeur sur la composition des troupeaux et leur orientation stratégique. Cette évolution du contexte sanitaire va infléchir les trajectoires des élevages selon différentes voies d'intensification dans un contexte fortement concurrentiel.

Les indicateurs de capacités d'innovation dégagés par cette étude seront utilisés pour suivre quantitativement les différents scénarios d'évolution prenant en compte les logiques d'action des éleveurs afin d'évaluer l'impact socioéconomique de l'éradication des populations de glossines dans cette région. La méthodologie présentée est utilisable pour comprendre l'impact du contrôle d'autres infections à transmission vectorielle sur les dynamiques d'innovation des éleveurs.

\section{Mots-clés}

Analyse compréhensive - Étude de cas - Intensification écologique - Maladie à transmission vectorielle - Race bovine - Risque sanitaire - Sénégal - Système d'élevage - Théorie de l'acteur-réseau - Trypanosomose animale africaine - Zone des Niayes. 


\section{Introduction}

En Afrique sub-saharienne, les conditions agro-écologiques et les systèmes d'élevage sont tels que les infections à transmission vectorielle représentent une contrainte à l'intensification des productions animales chez les ruminants. Les trypanosomoses animales africaines transmises par les glossines (Diptera, Glossinidae), ou nagana, sont ainsi considérées comme la principale contrainte sanitaire à l'intensification de l'élevage bovin (1). Leur impact varie selon les systèmes de production et les capacités à mettre en place une stratégie de lutte intégrée, qui nécessite généralement d'innover en réalisant des investissements et en changeant les pratiques d'élevage (2).

Au Sénégal, un projet d'éradication d'une population de glossines (Glossina palpalis gambiensis) a été mis en place dans la région côtière des Niayes, bande de 30 à 40 km située le long de la côte Atlantique, où des conditions micro-climatiques particulières sont propices à l'intensification de l'agriculture et de l'élevage. Ce projet est coordonné par la Direction des Services vétérinaires, avec un objectif d'intensification écologique (3) : il s'agit en effet d'augmenter la production et la productivité de l'élevage bovin tout en réduisant la population bovine. Les auteurs ont étudié l'impact de la levée de la contrainte sanitaire (trypanosomoses animales africaines) associée à l'éradication de cette population de vecteurs selon les systèmes de production. L'hypothèse est que la disparition du risque trypanosomien a d'une part un effet positif sur la productivité des systèmes d'élevage bovin et d'autre part influence les trajectoires d'innovation en favorisant le changement de régime socio-technique (groupes d'actants structurant l'innovation technologique et habitudes cognitives associées) (4). Comprendre ces trajectoires est un défi important pour quantifier les bénéfices socio-économiques associés à cette intervention et nécessite d'analyser les dynamiques à l'échelle du réseau socio-technique.

\section{Méthodes}

L'évolution des stratégies des éleveurs et de leurs pratiques a été étudiée grâce à l'analyse croisée de dix études de cas basées sur des entretiens compréhensifs (quatre entretiens par éleveur espacés d'au moins une semaine). Des outils d'épidémiologie participative ont été utilisés pour aborder avec les éleveurs le contexte sanitaire. Les éleveurs ont été sélectionnés aléatoirement dans la zone ciblée par ce projet après stratification de leur population (environ 1000 éleveurs dont 513 géolocalisés lors d'une enquête préliminaire) sur les systèmes d'élevage préalablement identifiés par une étude socio-économique (3) : trois éleveurs utilisant majoritairement des races exotiques (correspondant à $17 \%$ des éleveurs dans la zone d'étude), deux éleveurs utilisant principalement des Gobras (18 \%) et cinq éleveurs utilisant principalement des bovins trypanotolérants (65\%).

\section{Approche globale des stratégies d'exploitation par entretiens compréhensifs}

Les études de cas ont été utilisées afin d'identifier de nouveaux processus et concepts (5). Il s'agit de renforcer une boucle récursive abduction / déduction / induction (Fig. 1) (6). Les pratiques des éleveurs traduisent leur rationalité, portant à la fois sur ce que sont les choses et sur ce qui est souhaitable (normes sociales). Pour comprendre une action, il est nécessaire de connaître ce qui l'explique et la justifie pour les sujets eux-mêmes (7). Ainsi, les pratiques ont été reliées aux conceptions qui leur sont associées, en étudiant la manière dont elles sont élaborées, afin de comprendre les dynamiques en cours (8). En effet, les processus décisionnels du quotidien sont issus de formes collectives de pensée, remodelées en fonction des débats qui ont lieu dans le réseau de dialogue des sujets, et intègrent la gestion du risque.

Les hypothèses et liens de causalité identifiés dans cette phase inductive seront ultérieurement explorés par une enquête socio-économique basée sur un échantillonnage plus large (composante hypothético-déductive, Fig. 1).

\section{Utilisation des outils de l'épidémiologie participative}

Le risque trypanosomien est très hétérogène dans les Niayes, mais il existe des données précises sur la répartition spatiale des vecteurs cycliques des trypanosomoses animales africaines (9).

Pour évaluer la perception du risque de maladies vectorielles, chaque éleveur a réalisé une matrice de notation des maladies afin d'adopter un langage commun sur les maladies et de récolter les connaissances et perceptions spécifiques aux trypanosomoses animales africaines (10, 11). Des empilements proportionnels ont été réalisés sur l'incidence annuelle des trypanosomoses animales africaines depuis 2010. Ces données semi-quantitatives ont été croisées avec les données quantitatives des traitements trypanocides et autres administrés en 2010 et 2013.

Les races ont été classées par les éleveurs selon les trois critères les plus récurrents lors des descriptions (esthétique, productivité, rusticité), après une phase d'entretiens compréhensifs.

\section{Analyse des réseaux socio-techniques et de dialogue}

Les conceptions individuelles qui sous-tendent l'action sont issues d'une construction collective et l'usage des 


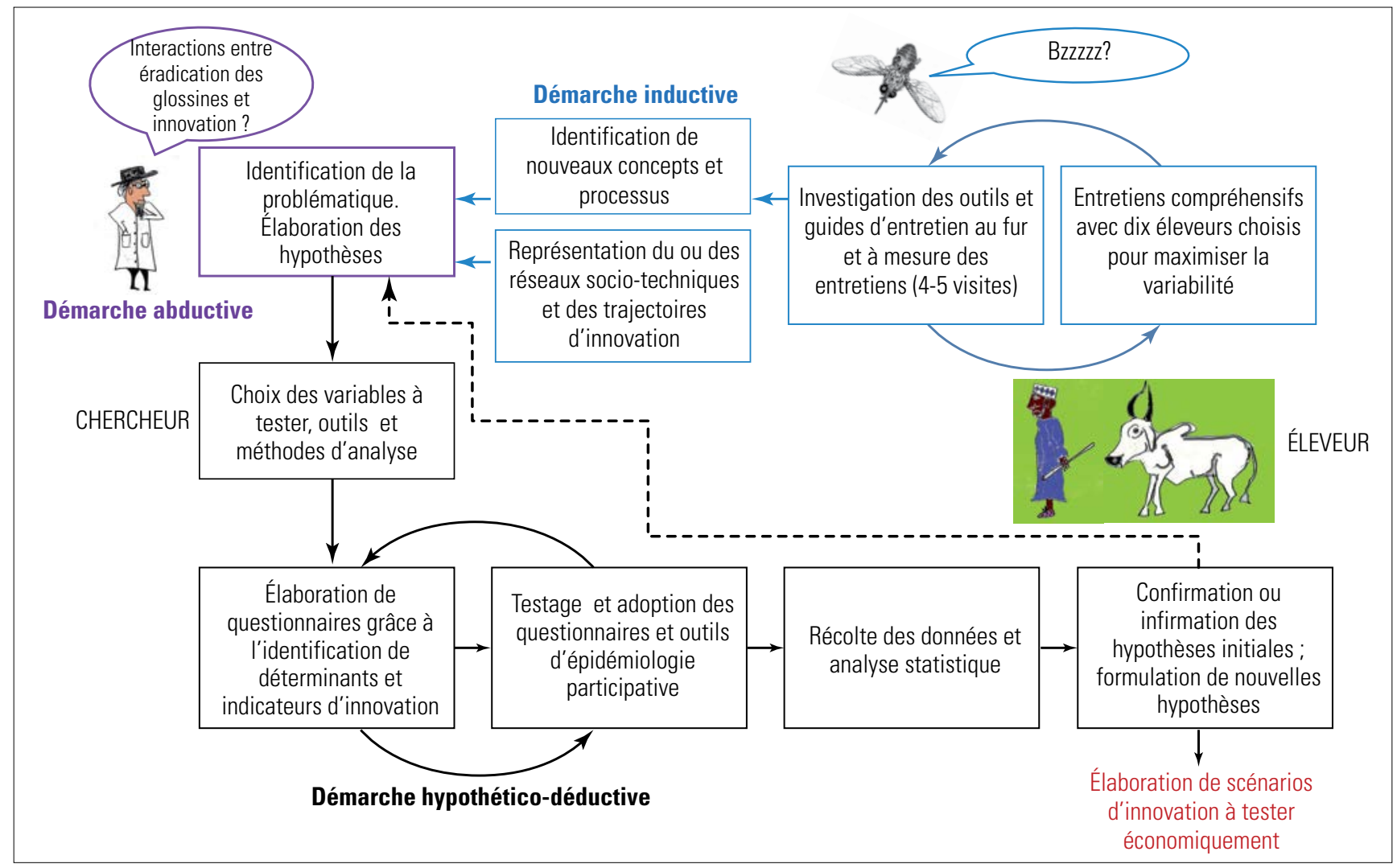

Déduction : tirer une conséquence à partir d'une règle générale et d'une observation empirique (cas)

Induction : trouver une règle générale qui pourrait rendre compte de la conséquence si l'observation empirique est vraie

Abduction : élaborer une observation empirique qui relie une règle générale à une conséquence (retrouver la conséquence si la règle est vraie)

\section{Fig. 1}

Démarche méthodologique adoptée pour caractériser les interactions entre risque trypanosomien et systèmes d'élevage dans les Niayes

Les résultats présentés dans cette publication correspondent à la démarche inductive

technologies et des innovations passe par la sollicitation d'un réseau socio-technique $(12,13)$. L'étude des dynamiques a ainsi nécessité un changement d'échelle, de l'exploitation agricole aux réseaux socio-techniques dans lesquels elle s'insère. Pour cela, l'analyse s'est inscrite dans le cadre méthodologique de la sociologie de l'acteur-réseau (14), qui envisage la contribution des entités humaines et non humaines de la même manière (actants). Les groupes sociaux et les réseaux de dialogue ont été caractérisés, car la diversité des relations et des débats (avec les pairs, les partenaires techniques...) favorise l'innovation.

\section{Résultats}

\section{Coexistence de trois systèmes d'élevage dans les Niayes}

Les trajectoires des élevages associés aux objectifs personnels des éleveurs permettent de caractériser les points de vue objectivement situés (8) et de comprendre les trajectoires d'innovation. Ainsi, la typologie employée a été modifiée par rapport à celle qui avait été utilisée pour l'échantillonnage. La première dichotomie est observée entre les producteurs laitiers ( $n=2 / 10)$, qui ont commencé leur activité d'élevage avec des fonds provenant d'une autre activité rémunératrice et qui ont acquis par achat des bovins de race exotique laitière, maintenus en stabulation permanente, et les éleveurs possédant des troupeaux allant au pâturage $(n=8 / 10)$. Parmi ces derniers, on distingue les pasteurs et agro-pasteurs $(n=6 / 8)$, qui possèdent des troupeaux à effectif moyen à grand dont la vocation est principalement bouchère, des agro-éleveurs ( $n=2 / 8$ ) qui, initialement agriculteurs, ont développé des troupeaux petits à moyens dans une visée de production mixte viande et lait.

Leséleveurs laitiers ont réalisé des investissements importants en achetant des génisses gestantes en France, en pratiquant l'insémination artificielle en routine et en s'équipant avec des infrastructures et équipements modernes. Ils recourent aux services vétérinaires privés et publics et à des prestataires de service en France pour la génétique et la santé animale 


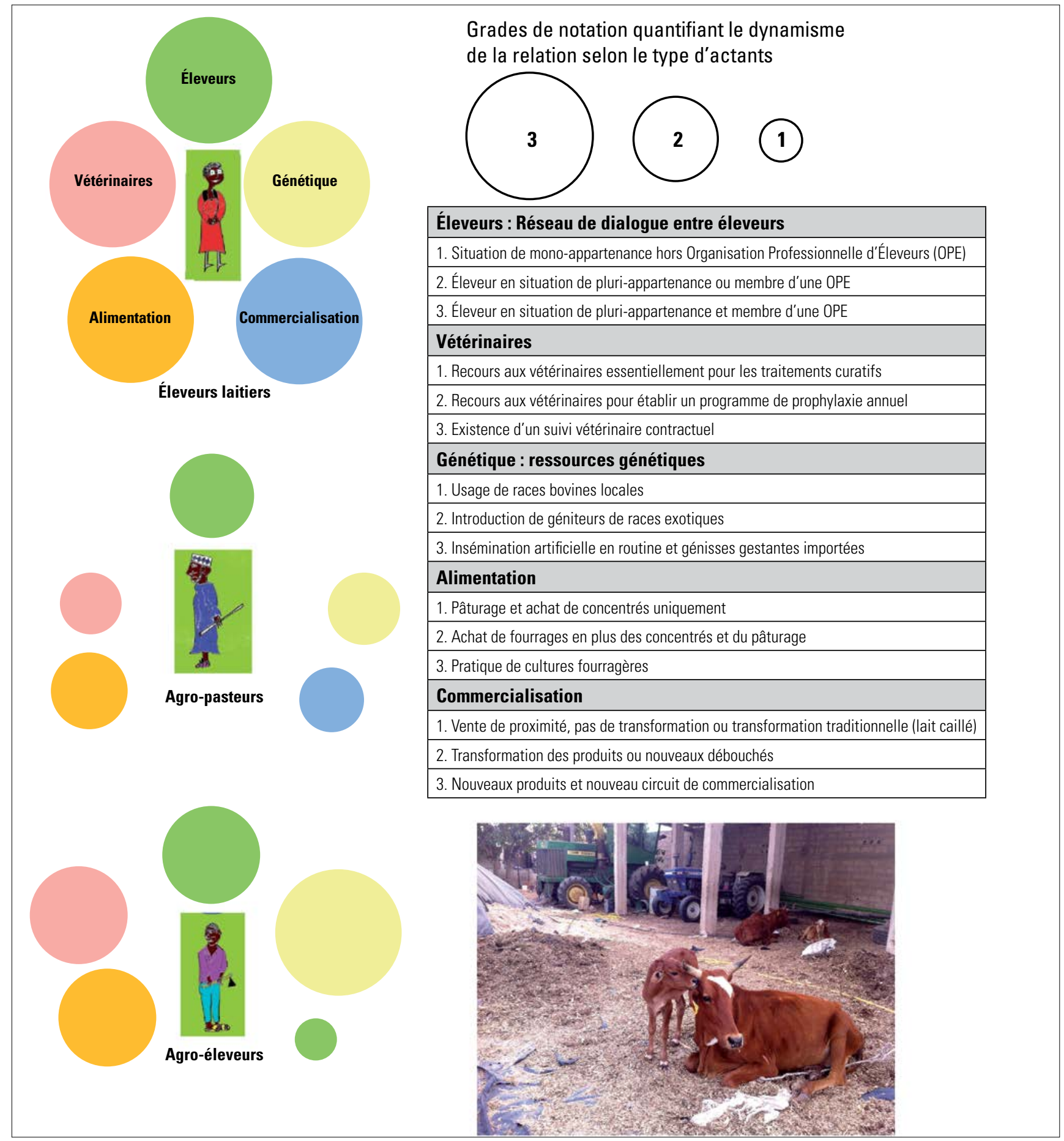

Fig. 2

Relations des éleveurs avec les principaux groupes d'actants du réseau socio-technique de l'élevage dans les Niayes en fonction du système d'élevage

La distance entre les boîtes (éleveurs) et les bulles (actants) est proportionnelle à la fréquence des contacts ; la taille des bulles est proportionnelle au dynamisme de la relation vis-à-vis des capacités d'innovation

(Fig. 2). L'usage intensif d'intrants et la culture fourragère (maïs, sorgho) caractérisent ce système.

La majorité des pasteurs et agro-pasteurs interrogés, d'ethnie peuhle (majoritaire dans l'échantillon de 277 élevages enquêtés en 2010 [3]), recherchent dans leur activité d'élevage une adhésion à leur identité, notamment en possédant un troupeau tyossan ou diofndé aada pour « être un vrai éleveur » (Tableau I). Bien que la plupart aient une tradition d'élevage, certains élèvent un troupeau issu 
Tableau I

Désignation et sens de quelques termes en wolof et pulaar utilisés par les éleveurs

\begin{tabular}{|c|c|c|}
\hline Termes & Traduction/désignation & Sens pour les usagers \\
\hline Nagé, nayi & $\begin{array}{l}\text { Nagé: « vache » au singulier en pulaar (nayi, au } \\
\text { pluriel) }\end{array}$ & Les bovins de manière générale \\
\hline Tyossan & "Tradition » en wolof & $\begin{array}{l}\text { Le troupeau tyossan est le troupeau d'origine, qui est transmis de génération en génération } \\
\text { et qui a un rôle de transmission des lignées ancestrales de bovins à l'intérieur de la famille }\end{array}$ \\
\hline Diofndé aada & « Parc de nuit traditionnel » en pulaar & Par extension, le troupeau traditionnel, qui est l'équivalent du troupeau tyossan \\
\hline Nayi wuro & "Vaches de la maison/ de la ville » en pulaar & $\begin{array}{l}\text { Ce sont des vaches achetées pour la reproduction et qui ne sont pas vendues. L'éleveur } \\
\text { peut élever la race de son choix }\end{array}$ \\
\hline Thiogal & «Grand troupeau » en pulaar & $\begin{array}{l}\text { Désigne les taureaux achetés dans les systèmes pastoraux traditionnels (hors des Niayes) } \\
\text { et revendus pour l'embouche }\end{array}$ \\
\hline Moussane & "Épuisement » en wolof & $\begin{array}{l}\text { Similaire aux trypanosomoses animales africaines dans les matrices de notation ; fait le lit } \\
\text { des autres maladies }\end{array}$ \\
\hline Sompt, somptou & L'équivalent de moussane en pulaar & Similaire aux trypanosomoses animales africaines dans les matrices de notation \\
\hline Ripass & "Aliment bétail » en wolof & L'aliment bétail en granulés \\
\hline Diambours & "Personnes neutres » en wolof & $\begin{array}{l}\text { Personnes choisies pour leur neutralité vis-à-vis des protagonistes et sollicitées pour } \\
\text { arbitrer un conflit à l'amiable }\end{array}$ \\
\hline Loumas & "Marchés hebdomadaires » en wolof & $\begin{array}{l}\text { Marchés à bestiaux traditionnels hebdomadaires ; désignés souvent par la ville où le } \\
\text { marché à lieu }\end{array}$ \\
\hline Gobour & La race Gobra en pulaar, appelée aussi peuhl-peuhl & Le zébu peuhl du Sénégal \\
\hline
\end{tabular}

\section{Tableau II}

Présentation des modes d'alimentation des troupeaux observés sur les dix études de cas selon les systèmes d'élevage

\begin{tabular}{|c|c|c|c|}
\hline $\begin{array}{l}\text { Système } \\
\text { d'élevage }\end{array}$ & Pâturage & Résidus de récolte & Concentrés \\
\hline Laitier & $\begin{array}{l}\text { Les animaux sont en stabulation } \\
\text { permanente pour un meilleur contrôle } \\
\text { des conditions sanitaires et pour } \\
\text { faciliter la traite deux fois par jour }\end{array}$ & $\begin{array}{l}\text { Certains éleveurs ne faisant pas partie de } \\
\text { l'échantillon de cette étude achètent des résidus de } \\
\text { maïs ou de haricot. La paille est achetée hors des } \\
\text { Niayes }\end{array}$ & $\begin{array}{l}\text { Choisis en fonction du meilleur rapport qualité/prix } \\
\text { et donnés selon les besoins estimés par calcul par } \\
\text { lot (stade physiologique) }\end{array}$ \\
\hline Agro-éleveur & $\begin{array}{l}\text { En fonction de la localisation, la } \\
\text { période de pâturage varie de } 12 \text { à } \\
4 \text { mois lorsqu'il s'agit d'éviter les } \\
\text { dégâts aux cultures }\end{array}$ & $\begin{array}{l}\text { Donnés à partir des champs des propriétaires } \\
\text { ou négociés avec les voisins au meilleur prix. } \\
\text { Complémentation nouvelle par les cartons }\end{array}$ & $\begin{array}{l}\text { Donnés préférentiellement aux bovins à } \\
\text { l'engraissement ou en lactation, sans calcul de } \\
\text { ration et quelquefois de manière irrégulière en } \\
\text { relation avec les opportunités d'achat }\end{array}$ \\
\hline Agro-pasteur & $\begin{array}{l}\text { Le pâturage est pratiqué toute } \\
\text { l'année. En fin de saison sèche, les } \\
\text { bergers utilisent les fourrages ligneux }\end{array}$ & $\begin{array}{l}\text { Usage très variable ; toxicité versus recherche de } \\
\text { qualité (selon l'impact sur la production). Négociés, } \\
\text { pendant la deuxième partie de la saison sèche, } \\
\text { collectivement lorsqu'il s'agit des champs des agro- } \\
\text { industriels }\end{array}$ & $\begin{array}{l}\text { Ils sont donnés préférentiellement aux animaux } \\
\text { à l'engraissement ou en lactation, sans calcul de } \\
\text { ration et souvent sur une période restreinte à la fin } \\
\text { de la saison sèche }\end{array}$ \\
\hline
\end{tabular}

d'animaux hérités, et d'autres ont reconstitué un troupeau «nayi wuro » qui redeviendra un troupeau tyossan pour leurs enfants (Tableau I). Ces troupeaux ont pour vocation de perpétuer des lignées familiales, ancestrales de bovins (15): ils sont l'objet de normes de transmission (par héritage) et de gestion zootechnique spécifiques. Ces lignées appartiennent aux races zébu Gobra et Djakoré (métisse entre Gobra et N'Dama, cette dernière race étant trypanotolérante) et les acquisitions de bovins se réalisent au marché hebdomadaire traditionnel (louma). La norme sociale de l'élevage pastoral peuhl se caractérise à l'origine par de faibles investissements en infrastructure et en matériel ainsi que par l'exploitation des ressources naturelles par le pâturage et la transhumance saisonnière, les dépenses liées à la complémentation étant restreintes à la fin de saison sèche. Chez les éleveurs enquêtés, une tendance à l'augmentation des dépenses alimentaires a été néanmoins observée, en relation avec les difficultés d'accès aux ressources naturelles et l'élevage de nouvelles races (Tableau II).

Des modes d'allotement des bovins très contrastés ont été observés. Chez les éleveurs laitiers, il y a d'une part les 
lots de génisses et de vaches de races européennes (dont les proportions par races sont justifiées techniquement) et d'autre part des lots de vaches de races locales (Djakoré, zébus Gobra ou Maure) ou des métisses. Cette stratégie est justifiée par le fait de garder des vaches plus rustiques pour limiter la baisse de la production laitière pendant les mois les plus chauds.

Chez les agro-pasteurs ( $n=6 / 8)$ on observe soit l'existence d'un unique troupeau avec reproduction (tyossan ou reconstitué), soit la séparation entre un lot tyossan, un lot de bovins d'embouche et quelquefois un lot de bovins en confiage (bovins appartenant à des bouchers). Dans un contexte urbain/périurbain, on observe une réduction des effectifs de certains troupeaux tyossan bien que la norme sociale soit de les augmenter. Diverses contraintes, en plus de la diminution des pâturages naturels, sont énoncées selon la localisation des élevages : la crainte de cas de mortalité subite dus à l'ingestion de sachets plastiques, la crainte des vols d'animaux de grand gabarit, ou les tensions avec le voisinage à cause des nuisances. L'élevage et le commerce des taureaux d'embouche nés hors des Niayes (thiogal) permettent de répondre aux besoins économiques avec une durée d'élevage réduite car les bovins sont achetés adultes sur les marchés, selon des critères différents des animaux reproducteurs.

On observe plusieurs variantes des normes sociales concernant la gestion de la reproduction dans les troupeaux tyossan alors que les normes de transmission (héritage) sont davantage conservées: les éleveurs évoquent à ce propos les prescriptions religieuses. En effet, alors que certains éleveurs évoquent la règle ancienne selon laquelle les géniteurs mâles doivent être obligatoirement nés dans le troupeau, certains introduisent des géniteurs de race différente (de phénotype proche) et d'autres pratiquent même l'insémination artificielle.

\section{Lien entre perceptions des races et systèmes d'élevage}

Les agro-pasteurs et pasteurs $(n=6 / 10)$ apprécient les zébus de race Gobra pour des raisons culturelles et parce que cette race représente un bon compromis entre esthétique, productivité et rusticité (Fig. 3). Appelée gobour ou, de manière assez explicite, peuhl-peuhl, elle est la seule race à avoir fait l'objet d'une description assimilable à un standard de race (Encadré 1). Cette race est valorisée pour son grand format, sa rusticité et la qualité de son lait, riche en matières grasses (Encadré 1). Les éleveurs préfèrent rester dans le standard de race pour le troupeau tyossan et diversifier les races au niveau des taureaux d'embouche. Pour les agroéleveurs et les éleveurs laitiers enquêtés, c'est aussi la race préférée pour le métissage. Ces animaux sont très sensibles aux trypanosomoses animales africaines (16).
Les Djakorés, appelés parfois N’Dama ou nago ordinaire, sont appréciés de manière globale pour leur rusticité et leur longue carrière de reproduction, à la fois par les agroéleveurs et les agro-pasteurs (Fig. 3). Certains l'assimilent à la race N'Dama. Un agro-pasteur qui, malgré un risque trypanosomien élevé, a diversifié les races grâce à une prophylaxie médicale rigoureuse indique que ses femelles N'Dama sont celles qui ont la plus grande longévité et le plus grand nombre de vêlages. La race descend dans les classements en termes d'esthétique, de productivité et même de rusticité lorsque le risque trypanosomien diminue (hors zone à glossines). Des métissages non spécifiquement Djakorés sont observés par introduction de géniteurs de races variées et par insémination, quelquefois pratiquée dans des troupeaux hérités.

Les races européennes sont perçues très différemment selon les systèmes d'élevage (Fig. 3). Le réseau d'éleveurs intensifs a développé des savoirs spécifiques aux différentes races dans le contexte local alors que les autres éleveurs essaient les différentes races sans préférence spécifique (les métis sont appelés «nago pompés »). L'une des recommandations qui circulent au sein de l'association des éleveurs intensifs laitiers préconise une composition de troupeau associant deux tiers de Montbéliarde ou Jersiaise pour la qualité du lait à un tiers de Holstein pour la quantité de lait. La race Holstein est décrite comme la plus productive par lactation, mais pas sur l'ensemble de la carrière en raison d'une longévité moindre. Le choix des races ainsi que de la taille idéale des troupeaux est toujours l'objet de débats dans ce réseau, influencés par les expériences locales et les liens commerciaux.

Les agro-éleveurs placent les races européennes plus haut dans leur classement que les agro-pasteurs, en relation avec de meilleurs apports fourragers. Ceux qui ont essayé l'insémination trouvent que les métis F1 ne sont pas assez « résistants » et présentent une moins bonne conformation lorsqu'ils vont au pâturage ; ils voudraient donc améliorer le métissage. Ils ont pourtant augmenté leurs dépenses de santé en se rapprochant des vétérinaires par la mise en place d'une meilleure prophylaxie sanitaire.

Concernant les races locales, les éleveurs enquêtés expriment une préférence pour les veaux nourris sous la mère pour obtenir des géniteurs en meilleure santé et n'envisagent donc qu'une commercialisation partielle de la production laitière (voir Encadré 1). Enfin, la race Guzérat (race exotique importée et diffusée par l'État sénégalais dans les années 60 et 70) a une très bonne réputation parmi les agro-pasteurs et agro-éleveurs, qui apprécient son esthétique (proche de la Gobra) et sa productivité mais expriment des difficultés à trouver des reproducteurs. La race Pakistanaise est préférée pour la production laitière. 

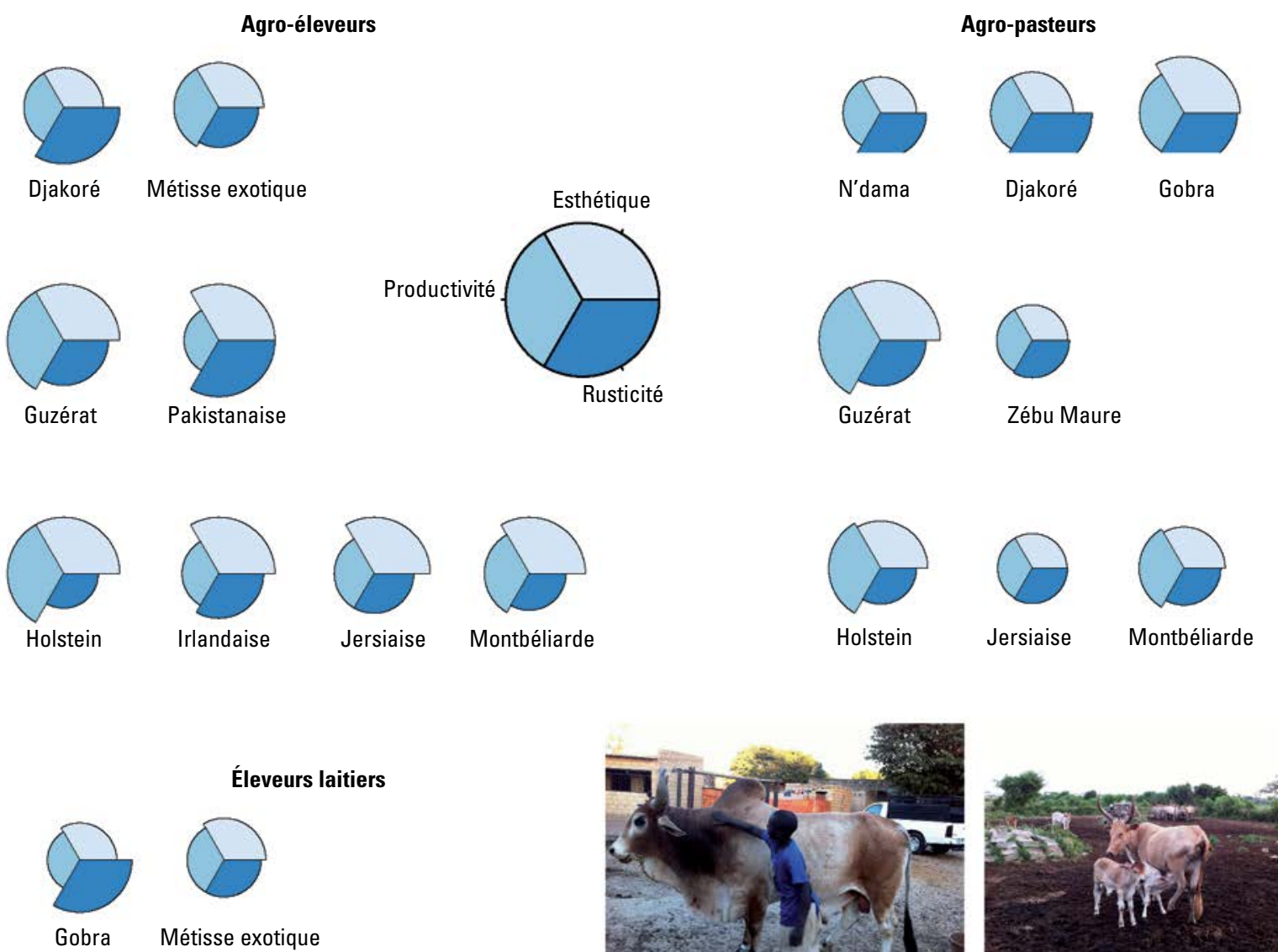

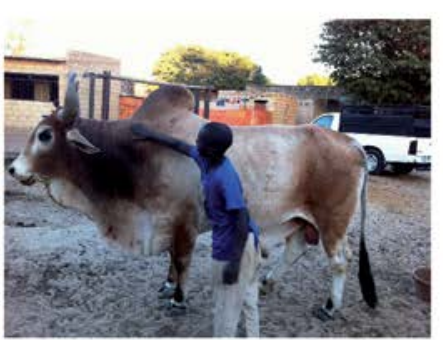

Guzérat

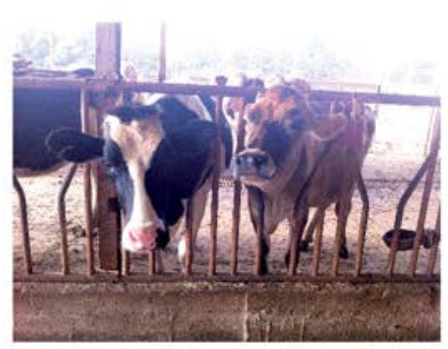

Holstein (G) \& Jersiaise (D)

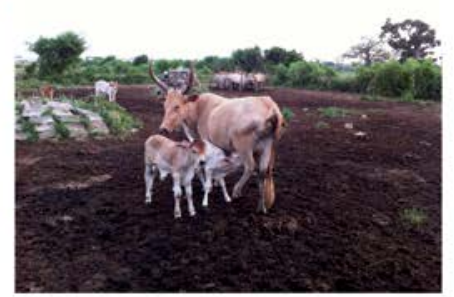

Djakoré

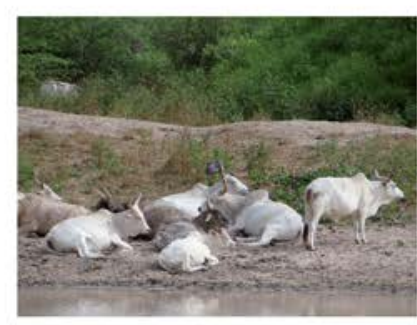

Gobra

Fig. 3

Perceptions des races bovines par les éleveurs selon les systèmes d'élevage

Les éleveurs ont classé les races selon trois critères : I'esthétique, la productivité et la rusticité. Les quatre premières races ont été gardées par classement et les races non classées pour un paramètre bien que citées pour un autre paramètre ont un classement correspondant à un score de 5 . Les points attribués sont inversement proportionnels à la place dans le classement (par paramètre) et une moyenne a été réalisée par type d'éleveurs pour construire les radars.

\section{Site d'implantation des élevages, exposition au risque et perception du risque}

La distribution des systèmes d'élevage est fortement liée à celle du vecteur : $34 \%$ des élevages situés dans la zone sans glossines utilisent majoritairement des races trypanosensibles contre $4 \%$ dans la zone avec glossines (3). Le fait que le risque trypanosomien soit trois fois moins élevé dans la zone sans glossines (17) a ainsi permis l'innovation au niveau des races. De plus, les éleveurs laitiers intensifs ont, dès leur installation, employé des docteurs vétérinaires comme gérants de ferme ou comme prestataires permanents. 


\section{Encadré 1}

\section{Extraits d'entretiens compréhensifs réalisés avec dix éleveurs dans la zone des Niayes, Sénégal en 2013 et 2014}

"Le gobour [Gobra] est blanc, il est très joli ! [...] En dehors de la couleur, l'animal présente une longue queue, un fanon développé ! II a de grandes cornes et une grande bosse ! "

(M.S., pasteur et commerçant de bovins à Thiès)

"Le gobour [Gobra] est la race préférée. Quand c'est bien nourri, il bat tout le monde ! II est plus résistant, à la chaleur, à la sécheresse. II supporte aussi la soif ! II peut rester une journée sans boire."

(M.S., pasteur et commerçant de bovins à Thiès)

"Mon berger, il ne fait la traite que le soir, il laisse les veaux téter la nuit et le matin pour qu'ils grandissent plus vite... "

(M.B., agro-éleveur à Niacoulrab)

"Le fait que l'animal a bien tété, qu'il a été bien nourri avec le lait, je vais le savoir. S'il n'a pas été bien nourri dans son enfance, il ne sera jamais bien. Je le sais grâce à mon expérience. Je regarde la base des cornes, le front !"

(M.S., pasteur et commerçant de bovins à Thiès)

"La Djakoré c'est une race adaptée, qui restait dans la zone. Avec l'avancée de la médecine vétérinaire, on arrive à élever d'autres races : la Mauresque, la Gobra, les métis. "

(M.B., agro-pasteur et boucher à Ndiakhirate-Digue)

"Le gobour [Gobra] est habitué à la terre d'ici. "

(M.D., agro-pasteur à Thiès)

"Sielle va en brousse, elle aura forcémentsompt[trypanosomoses animales africaines]. "

(M.S., agro-pasteur à Diacksaw Peuhl (Sangalkam)

"On ne le [somptou / trypanosomoses animales africaines] voit pas sur nos animaux car nous on traite. C'est fréquent dans la zone des Niayes "; " Elle [la maladie] tue rarement. Elle freine le développement de l'animal mais ne tue pas vite. II y a moins de lait."

(M.B., agro-pasteur et boucher à Ndiakhirate-Digue)
" Nous souhaitons développer l'agriculture et l'élevage. L'agriculture et l'élevage vont ensemble. "

(M.K., agro-éleveur à Kayar)

"L'agriculture et l'élevage sont liés, il faut les associer. "

(M.B., agro-éleveur à Niacoulrab)

"Les races locales sont plus résistantes ici mais elles sortent moins de lait ! L'État a donné des races pakistanaises, c'est très demandé actuellement. Non, ce n'est pas Guzérat. Je n'aime pas les Guzérats. C'est une race de viande, je cherche le lait. Le lait est vendu le soir même, à 500 francs CFA en gros à Keur Massar. Je le vends à 500 francs à ma femme et elle le revend à 600 francs. "

(M.B., agro-éleveur à Niacoulrab)

"Le fait d'être en GIE [groupement d'intérêt économique] est un atout car l'État reconnaît les groupements. Des gens veulent faire de l'insémination mais s'ils ne sont pas en GIE, c'est difficile."

(M.K., agro-éleveur à Kayar)

"Le métissage... dans le cadre d'améliorer la race. On nous a fait savoir que l'entretien de ces animaux n'était pas facile. II faudrait faire de la culture fourragère et donner du ripass et des résidus de culture en plus."

(M.K., agro-éleveur à Kayar)

" J'ai commencé avec la race locale, mais on m'a fait savoir que les animaux issus de l'insémination sont plus rentables parce qu'ils produisent plus de lait. Même à la vente, ils rapportent beaucoup plus que la race locale. Le Guzérat, lui aussi rapporte beaucoup d'argent car il est de grand gabarit. S'il n'y a pas ces deux races-là [métis et Guzérat], je choisis la race locale. "

(M.K., agro-éleveur à Kayar)
Ceux-ci échangent très fréquemment des informations entre eux via l'association et par des échanges informels (Fig. 2).

Les agro-éleveurs et agro-pasteurs enquêtés justifient le fait de démarrer leur élevage avec la race locale (à savoir, dans les Niayes, la race Djakoré) par la disponibilité et l'adaptation de celle-ci au contexte sanitaire. La contrainte des trypanosomoses animales africaines n'est pas explicitement citée comme critère de choix car les éleveurs ont une approche globale de la rusticité. Ils indiquent cependant que les races trypanosensibles sont moins adaptées au contexte sanitaire (Encadré 1).
Les éleveurs peuhls originaires du Nord du Sénégal ( $n=2 / 10$ ) préfèrent pour des raisons culturelles reconstituer leurs troupeaux avec la race Gobra : ils résident principalement dans la zone sans glossines (région de Thiès). Dans ce contexte sanitaire, c'est la race Gobra qui est citée comme la plus rustique et résistante aux maladies, notamment du fait de sa résistance à la dermatophilose (gaye ou ram) associée à la présence des tiques (Amblyomma variegatum [Acari, Ixodidae], diagnose non proposée) (Encadré 1).

Dans les matrices de notation, les tableaux cliniques appelés moussane ou sompt sont similaires aux trypanosomoses 
animales africaines et la description des signes cliniques est plus complète chez les agro-pasteurs ayant une tradition d'élevage que chez les agro-éleveurs et ceux qui utilisent la prophylaxie médicale. Une confusion serait cependant possible avec certaines infections transmises par les tiques (anaplasmose, babésiose). Le classement des contraintes sanitaires par les éleveurs fait apparaître des différences de perception du risque selon leur exposition au risque sanitaire : les cinq éleveurs dans la zone avec glossines citent les trypanosomoses animales africaines parmi leurs principales contraintes sanitaires et trois d'entre eux la citent en première place, contrairement aux éleveurs hors zone à glossines dont trois ne les citent pas du tout, alors que les races trypanosensibles qu'ils possèdent sont sous prophylaxie par trypanocides.

La diminution récente du risque liée au programme d'éradication des glossines n'est perçue que par les éleveurs de la zone à glossines élevant des Djakorés et n'utilisant pas de traitements prophylactiques : ils indiquent une forte diminution d'animaux atteints l'année suivant l'éradication, tout en s'abstenant de pronostic sur le futur.

Ainsi, les trypanosomoses animales africaines constituent une contrainte perçue par tous les éleveurs de notre échantillon. Tous utilisent des traitements trypanocides en curatif et la moitié appliquent les traitements prophylactiques deux à quatre fois par an, même dans la zone sans glossines. Cependant, les éleveurs ne présentent pas les trypanosomoses animales africaines comme une préoccupation actuelle, contrairement aux maladies telles que la dermatose nodulaire contagieuse bovine, la fièvre aphteuse ou la fièvre de la vallée du Rift, dont les incidences annuelles sont fortement variables (les avortements dus à la fièvre de la vallée du Rift étant une préoccupation surtout pour les éleveurs laitiers). Les éleveurs ne parlent pas du projet d'éradication des glossines. Ils ont intégré la gestion du risque trypanosomien dans leur conduite d'élevage, soit par la génétique trypanotolérante, soit par la prophylaxie médicale, soit les deux. Le contrôle des trypanosomoses animales africaines uniquement par les traitements curatifs n'est observé que chez les troupeaux Gobras, peu ou pas exposés aux glossines, ou les troupeaux de Djakorés.

Ceux qui sont exposés à un risque trypanosomien important associent le risque à une caractéristique constitutive de l'environnement sur laquelle on ne peut agir (Encadré 1). Ceux qui utilisent les trypanocides en prophylaxie estiment contrôler ce risque grâce à cette stratégie de prévention, tout en ayant connaissance de son impact sur la productivité (Encadré 1).

\section{Stratégies d'élevage et gestion des ressources alimentaires}

Les différents modes de gestion de l'alimentation (Fig. 2, Tableau II) peuvent être caractérisés selon la conduite des animaux sur parcours naturel, la production de cultures fourragères, l'achat de concentrés et l'achat de résidus de récolte et autres fourrages (foin, paille).

La conduite sur parcours naturel est la norme chez les éleveurs traditionnels : seuls les éleveurs intensifs laitiers maintiennent leurs vaches de races européennes ou métisses en stabulation permanente. Un éleveur intensif qui envoyait son lot de vaches Djakorés en petite transhumance a décidé d'arrêter cette pratique qu'il associait à un taux élevé de mortalité, attribué à une mauvaise prise en charge des animaux atteints de dermatose nodulaire contagieuse bovine et de fièvre de la vallée du Rift, couplée à un déficit alimentaire.

Les troupeaux de Djakorés et Gobras vont le plus souvent ( $n=7 / 8)$ au pâturage toute l'année mais les éleveurs signalent de manière unanime les difficultés croissantes liées à la diminution des surfaces accessibles aux troupeaux. Les alternatives observées sont l'usage de deux sites de pâturage selon la saison (saison sèche / saison des pluies), distants de quelques kilomètres et l'élevage à l'attache pendant une période longue ( $>6$ mois) pour éviter les dégâts aux cultures dans les cuvettes maraîchères. La stratégie des agro-éleveurs se caractérise par des troupeaux de taille faible à modérée et par l'intensification d'une production mixte viande et lait (Encadré 1). L'usage des résidus de récolte est très variable. Il existe même une controverse sur la toxicité des résidus de récolte à cause des pesticides. Les efforts pour obtenir des résidus de récolte varient donc beaucoup : certains éleveurs qui font du maraîchage donnent leurs résidus de récolte et complètent en négociant à faible coût avec d'autres maraîchers alors que certains investissent des sommes importantes pendant la saison sèche pour négocier de manière collective l'accès à l'hectare de résidus de haricot ou de maïs auprès des agro-industriels. Les prix sont très variables et les stratégies diffèrent selon les éleveurs : la norme la plus ancienne est de dépenser le minimum afin de limiter les pertes en saison sèche, quitte à déplacer le troupeau « pour se rapprocher des tiges de mil qui sont deux fois moins chères ", mais on observe une autre variante, nouvelle chez les agro-pasteurs, qui est d'acheter des résidus plus chers en privilégiant l'effet observé sur la production : "c'est cher mais c'est de la qualité ».

Enfin, la pratique de cultures fourragères n'a été observée que chez les éleveurs laitiers dont l'objectif est de produire suffisamment d'ensilage pour couvrir les besoins annuels du troupeau. Le foin et la paille sont achetés hors des Niayes.

\section{L'accès variable aux prestations techniques}

Deux réseaux de prestation de soins et conseils vétérinaires sont observés : d'une part les agents d'État qui effectuent des soins vétérinaires en plus de leurs fonctions régaliennes et d'autre part les cliniques vétérinaires privées. La répartition 
de ces prestataires n'est pas homogène et alors que les éleveurs situés en zone urbaine (Thiès, Sébikotane, etc.) indiquent avoir le choix, le problème de la disponibilité des vétérinaires est récurrent lorsque les éleveurs sont éloignés des centres urbains.

La dernière campagne d'insémination artificielle a été engagée par l'État en 2009 avec des semences de races laitières européennes (18). Cette campagne avait pour but de favoriser l'élevage de métisses F1 à vocation laitière chez les éleveurs extensifs. Certains éleveurs expliquent s'être regroupés en groupements d'intérêt économique (GIE) ou en associations afin de pouvoir bénéficier de l'insémination artificielle (Encadré 1). L'information qui circule entre éleveurs est «qu'une vache [de race européenne] peut entretenir toute une famille ». Les éleveurs ont reçu le message technique que les métisses avaient besoin d'être élevées en stabulation et qu'elles étaient plus exigeantes que les Gobras ou Djakorés sur le plan alimentaire (Encadré 1). Ceux qui n'ont pas pratiqué l'insémination évoquent soit le statut tyossan de leur troupeau et leur préférence pour la race « élevée par [le] père et [le] grand-père », soit le fait qu'ils ne veulent pas dépenser davantage en alimentation. Parmi ceux qui ont déjà pratiqué l'insémination $(n=7 / 10)$, ceux qui continuent à la pratiquer sont les deux producteurs laitiers qui en ont un usage exclusif et ceux qui se sont engagés dans l'amélioration génétique pour une production mixte (les deux agro-éleveurs et un agro-pasteur). Pour ceux qui recourent à l'amélioration génétique, le risque lié à cette innovation est considéré acceptable grâce au paiement différé et subventionné par l'État de l'insémination et au fait que les métisses valent plus cher à la vente, même si elles sont vendues plus précocement que les Gobras ou les Djakorés (Encadré 1).

Les éleveurs extensifs évoquent leurs difficultés à élever de manière rentable les métisses à cause de l'investissement nécessaire pendant plusieurs années avant les premiers bénéfices (temps de gestation et d'élevage jusqu'à la première lactation). Les éleveurs ayant une autre source de revenus (agriculture, commerce, etc.) sont ceux qui arrivent à élever les métisses pour la production laitière. Un programme national d'aide à l'obtention de prêt pour l'élevage est en cours mais la question de l'accès au crédit reste en discussion.

\section{Discussion}

Les données existantes sur l'élevage dans la zone des Niayes représentent principalement le point de vue des zootechniciens (3, 18, 19, 20, 21). La démarche participative des entretiens compréhensifs utilisée ici a permis de restituer les logiques d'action du point de vue des éleveurs (22).

\section{Réseaux socio-techniques et capacités d'innovation}

La préoccupation récurrente chez tous les éleveurs est la pression foncière (20). Elle entraîne un accès réduit aux pâturages, des conflits entre éleveurs extensifs et agriculteurs, et une incertitude quant à l'accès aux terres cultivables pour ceux qui produisent leur fourrage.

Alors que certains développent des stratégies pour éviter les conflits avec les agriculteurs en maintenant leurs animaux à l'attache ou en pratiquant une transhumance à faible distance, d'autres continuent le pâturage à proximité bien qu'ils doivent payer des amendes en cas de dégâts infligés aux cultures par les animaux. Face à ces conflits avec les agriculteurs, plusieurs stratégies sont observées : le recours à des médiateurs (diambours), ou la négociation entre associations et producteurs agricoles concernant les modalités d'accès aux résidus de récolte.

Cependant, un nouveau phénomène préoccupe certains éleveurs : le manque d'accès aux résidus de récolte à cause de la concurrence avec les producteurs intensifs qui viennent des autres communes rurales pour négocier avec les agriculteurs.

Une des principales fonctions de l'association des éleveurs laitiers est son rôle de lobbying auprès du ministère de l'Élevage. Ainsi, ses principales préoccupations sont la sécurisation de l'alimentation et de la santé animales. En effet, les éleveurs ne possèdent pas de terres et subissent chaque année l'incertitude de l'accès aux terres à louer. De plus, le réseau socio-technique de l'innovation « cultures fourragères » doit être amélioré : les éleveurs n'ont pas accès à du matériel agricole de bonne qualité, les semences fourragères ne sont pas adaptées à leurs besoins... Enfin, en santé animale, ces élevages réclament un accès plus rapide aux vaccins suivant l'évolution du contexte sanitaire (épizooties de fièvre aphteuse, de dermatose nodulaire contagieuse bovine, de fièvre de la vallée du Rift...).

Le choix des races est aussi un sujet à débat fréquent entre éleveurs et avec les vétérinaires publics et privés : ces derniers sont très présents dans les réseaux de dialogue des éleveurs en voie d'intensification ou avec un degré important de pluri-appartenance (relations de dialogue avec des groupes sociaux différents) (Fig. 3). Les éleveurs les plus traditionnels se réfèrent essentiellement aux expériences de leurs pairs (leur réseau de dialogue est essentiellement circonscrit au groupe de producteurs localisé).

La commercialisation du lait est une préoccupation seulement pour les éleveurs intensifs qui doivent changer leurs pratiques de transformation et de distribution audelà d'un volume seuil. On observe qu'un nouveau réseau d'acteurs et de nouvelles normes de consommation sont en construction. 
Bien que le gouvernement ait joué un rôle incitatif important via ses campagnes d'insémination (23), le secteur privé a pris le relais et importe génisses gestantes et semences ainsi que les médicaments et matériel associés, quelquefois même au service de l'État. Les partenariats avec des coopératives et firmes européennes basés sur des relations commerciales s'accompagnent de flux d'informations techniques et donc d'apprentissages. La préférence d'une race par les éleveurs ne suffit pas à son succès (exemple : manque de disponibilité de reproducteurs Guzérat) : le réseau peine à se reconfigurer à son avantage alors que les races européennes sont favorisées par la circulation de semences pour insémination.

\section{Trajectoires d'innovation en relation avec la suppression du risque trypanosomien}

La suppression du risque trypanosomien améliore la productivité des races trypanosensibles et retire l'avantage de la trypanorésistance dans l'appréciation de la rusticité, mais aussi de la productivité et de l'esthétique des races. Cela va engager les éleveurs à abandonner les Djakorés pour des races trypanosensibles plus productives et mieux conformées dans un contexte indemne de trypanosomoses animales africaines (Fig. 3).

Les éleveurs de Gobras ne représentent que $3 \%$ des éleveurs dans la zone infestée, contre $21 \%$ dans la zone sans glossines. Étant donné que, pour chaque système d'élevage, la manière de solliciter le réseau socio-technique est similaire pour les races Djakoré (65\% des éleveurs globalement) et Gobra, le changement de l'une vers l'autre race est aisé. L'incertitude est plus grande concernant la transition vers l'élevage laitier. En 2004, les fermes laitières représentaient $1 \%$ des élevages bovins dans les Niayes (18). En 2010, cette proportion a atteint $19 \%$ dans la zone sans glossines contre $8 \%$ dans la zone infestée (3).

Les éleveurs laitiers sont résolument engagés dans l'innovation mais avec une prise de risque qui n'est possible que par l'apport important de fonds. Leurs systèmes de normes et leur réseau d'acteurs ne sont pas encore stabilisés.

Des savoirs et savoir-faire se développent au sujet de la zootechnie des races exotiques : ils s'élaborent à la fois dans le réseau des intensifs laitiers et chez les éleveurs extensifs, en particulier chez les agro-éleveurs, avec un flux d'informations entre les deux. Le degré de pluri-appartenance des éleveurs conditionne les flux d'informations et les comparaisons en fonction des conditions d'élevage.

Les normes de gestion du troupeau présentent de nombreuses variantes qui s'expliquent par la phase de transition entre régimes socio-techniques. Certains éleveurs extensifs posent la question de comment opérer cette transition techniquement et financièrement. En particulier, les éleveurs expérimentent les métissages avec peu d'encadrement technique pour combiner rusticité et productivité et produire leurs propres reproducteurs, alors que la politique du ministère prévoit un recours permanent à l'insémination. Les éleveurs intensifs posent les questions de comment sécuriser l'accès à la terre et comment développer la commercialisation. Globalement, tous les éleveurs sont confrontés au problème (à résoudre) de la sécurisation de leur accès au foncier. Cette contrainte est favorable à l'intensification et donc à l'innovation.

L'intégration agriculture-élevage est un concept investi par les agro-éleveurs (24), qui veulent une taille de troupeau moyenne à faible, en stabulation saisonnière ou permanente, en relation avec leurs capacités d'alimentation fourragère. Ils ont un objectif d'intensification mixte (lait et viande) basée sur des races exotiques, pour lequel l'acquisition des savoirs et savoir-faire n'en est qu'à ses débuts. L'éradication des glossines aura un effet facilitateur de la transition, qui pourrait continuer vers une intensification laitière.

Selon C. Corniaux, le lait est, dans les sociétés lignagères du Nord du Sénégal, un produit social dont la gestion est soumise à des règles sociales qui conditionnent la contrainte technique (25). Ici, les agro-pasteurs et pasteurs envisagent la commercialisation du lait comme une source de revenus additionnelle " pour gérer le quotidien » (dépenses alimentaires réalisées par les épouses) tout en gardant un objectif principal de production bouchère ; certains envisagent la stabulation d'un noyau de vaches laitières : c'est donc un indicateur à suivre. L'embouche et la commercialisation de races trypanosensibles comme les zébus Gobra et Guzérat constituent une voie probable d'intensification pour ces derniers car elles permettent de générer davantage de fonds, à taille de cheptel constant. Comme les réseaux d'acteurs et de normes sociales sont similaires, la transition sera aisée.

Une étude récente effectuée dans la région des Niayes a montré que dans les systèmes d'élevage utilisant des races trypanosensibles (Gobra ou exotique), la taille du troupeau était inférieure de $45 \%$ et les ventes annuelles des productions (lait et viande) étaient de $250 €$ (s.d. 513) par tête contre $74 €$ (s.d. 38) dans le système traditionnel trypanotolérant $\left(p<10^{-3}\right)$ (3). Les gains attendus de l'éradication des glossines reposent donc sur un changement du système d'élevage avec abandon de la race Djakoré, ce qui est cohérent avec les trajectoires envisagées. Cependant, la rentabilité financière dépend de la vitesse de transition des systèmes. À Zanzibar, un taux annuel de changement de système d'élevage de $2 \%$ a été observé les cinq premières années après l'éradication de Glossina austeni (26). Cependant, sachant que dans la région des Niayes, le réseau socio-technique de l'élevage est déjà en cours de reconfiguration avec le renforcement d'un réseau de nouveaux acteurs, de nouvelles normes et l'accumulation 
d'apprentissages, il est probable que la vitesse des transitions suivra la courbe en $\mathrm{S}$ de l'innovation : il y aura une accélération prononcée de celle-ci après la phase initiale d'adoption par les innovateurs précoces (27). Une enquête socio-zootechnique sur un échantillon plus large permettra de suivre les indicateurs de capacités d'innovation, à savoir : la typologie initiale de l'élevage (en particulier le statut des troupeaux), les capacités d'investissement (par crédit ou double activité), le mode d'alimentation fourragère des bovins, l'appartenance à une organisation professionnelle d'éleveurs (OPE), le mode de gestion de la reproduction, les races des géniteurs. Il sera alors possible d'améliorer le modèle économique grâce à des scénarios plus crédibles que ceux précédemment testés (3), ce qui est nécessaire pour estimer le ratio coûts-bénéfices de l'intervention de lutte anti-vectorielle. Ainsi, entre un scénario à faible taux de transition annuel de $2 \%$ des éleveurs possédant des races trypanotolérantes vers les autres catégories d'éleveurs, et un scénario suivant une courbe en $\mathrm{S}$, les auteurs ont estimé une évolution du taux de rentabilité interne du projet de $10 \%$ à $19 \%$ respectivement (3).

L'analyse croisée d'études de cas a permis de comprendre comment le risque trypanosomien est intégré dans les logiques d'action des éleveurs dans les Niayes. L'évaluation de l'importance sanitaire et économique des maladies à transmission vectorielle ne suffit pas à mesurer l'impact d'un programme de lutte.

La démarche présentée ici peut être appliquée à d'autres infections à transmission vectorielle économiquement importantes et influentes sur les stratégies des éleveurs et les systèmes d'élevage. Dans cette même région des Niayes, on peut penser à la cowdriose transmise par Amblyomma variegatum, ou à la dermatophilose associée à la présence de cette même tique. Des méthodes intégrées de lutte contre ces maladies existent et pourraient faire l'objet d'innovations au Sénégal ou ailleurs $(28,29)$. D’une manière plus générale, il en va de même pour des infections non vectorielles, contre lesquelles des stratégies régionales et mondiales de contrôle progressif ou d'éradication sont en cours d'élaboration : une compréhension fine des dynamiques au sein des réseaux socio-techniques est essentielle pour favoriser l'innovation en matière de stratégies de contrôle, et pour évaluer leur impact socio-économique.

\title{
Importancia de las infecciones transmitidas por vectores según el sistema productivo: la tripanosomosis bovina y la dinámica de innovación de los ganaderos en el Senegal
}

\author{
F. Bouyer, J. Bouyer, M.T. Seck, B. Sall, A.H. Dicko, R. Lancelot \& E. Chia
}

\section{Resumen}

En una región del Senegal propicia a la intensificación de la producción ganadera, la región costera de los Niayes, se puso marcha un proyecto de erradicación de una población de glosinas (Glossina palpalis gambiensis) con el fin de eliminar la limitación que supone la tripanosomosis y hacer así posible una intensificación ecológica de la producción bovina.

El análisis cruzado de diez estudios de caso constituye la fase inductiva para evaluar la influencia que tendría la desaparición de esa limitación sanitaria sobre la trayectoria de las estrategias productivas. Para ello se apeló a los métodos del análisis exhaustivo y a las herramientas de la epidemiología participativa con objeto de aprehender las lógicas de trabajo. Se analizaron así las estrategias en función de los objetivos del ganadero y de su capacidad para movilizar a la red sociotécnica en el caso de tres grandes tipos de producción: la agropastoral, la agroganadera y la lechera intensiva.

El riesgo de tripanosomosis es una limitación que la praxis ganadera tiene integrada mediante el recurso a la genética tripanotolerante, la profilaxis médica 
o la elección de zonas de escaso riesgo para establecer explotaciones. Por ello la desaparición de ese riesgo tendrá una gran influencia en la composición de los rebaños y su orientación estratégica. Semejante evolución del contexto sanitario va a modificar la trayectoria de las explotaciones ganaderas, que seguirán diferentes vías de intensificación en un contexto de gran competencia.

Los indicadores de capacidad de innovación definidos a partir de este estudio serán utilizados para hacer un seguimiento cuantitativo de las diferentes hipótesis de evolución teniendo en cuenta las lógicas de trabajo de los ganaderos con el fin de evaluar la repercusión socioeconómica de la erradicación de las poblaciones de glosinas de la región. La metodología aquí presentada se puede utilizar también para saber cómo puede repercutir el control de otras infecciones transmitidas por vectores en las dinámicas de innovación de los ganaderos.

\section{Palabras clave}

Análisis exhaustivo - Enfermedad transmitida por vectores - Estudio de caso Intensificación ecológica - Raza bovina - Riesgo sanitario - Senegal - Sistema de producción - Teoría del del actor-red - Tripanosomosis animales africanas - Zona de los Niayes.

\section{Références}

1. Van den Bossche P., de La Rocque S., Hendrickx G. \& Bouyer J. (2010). - A changing environment and the epidemiology of tsetse-transmitted livestock trypanosomiasis. Trends Parasitol., 26 (5), 236-243.

2. Bouyer J., Bouyer F., Donadeu M., Rowan T. \& Napier G. (2013). - Community and farmer-based management of animal African trypanosomosis in cattle. Trends Parasitol., 29 (11), 519-522. doi:10.1016/j.pt.2013.08.003.

3. Bouyer F., Seck M.T., Dicko A., Sall B., Lo M., Vreysen M.J.B., Chia E., Bouyer J. \& Wane A. (2014). - Ex-ante benefit-cost analysis of the elimination of a Glossina palpalis gambiensis population in the Niayes of Senegal. PloS Negl. Trop. Dis., 8 (8), e3112. doi:10.1371/journal.pntd.0003112.

4. Geels F.W. \& Schot J. (2007). - Typology of sociotechnical transition pathways. Res. Policy, 36 (3), 399-417. doi:10.1016/j.respol.2007.01.003.

5. David A. (2003). - Études de cas et généralisation scientifique en sciences de gestion. Rev. Sci. Gestion, 39, 139-166. Page web: http://econpapers.repec.org/RePEc: dau:papers:123456789/1444 (consultée le 25 novembre 2014).
6. David A. (1999). - Logique, épistémologie et méthodologie en sciences de gestion. DRM Publications, Châtenay-Malabry, $23 \mathrm{pp}$.

7. Weber M. (1971). - Économie et société (J. Chavy \& E. Dampierre, trad.). Plon, Paris.

8. Ruault C. (1996). - L'invention collective de l'action : initiatives de groupes d'agriculteurs et développement local. L'Harmattan, Paris.

9. Dicko A.H., Lancelot R., Seck M.T., Guerrini L., Sall B., Lo M., Vreysen M.J.B., Lefrançois T., Williams F., Peck S.L. \& Bouyer J. (2014). - Using species distribution models to optimize vector control: the tsetse eradication campaign in Senegal. Proc. Natl Acad. Sci. USA, 111 (28), 10149-10154.

10. Catley A. (2000). - The use of participatory appraisal by veterinarians in Africa. Rev. Sci. Tech. Off. Int. Epiz., 19 (3), 702-719

11. Catley A., Alders R.G. \& Wood J.L.N. (2012). - Participatory epidemiology: approaches, methods, experiences. Vet J., 191 (2), 151-160. doi:10.1016/j.tvjl.2011.03.010. 
12. Akrich M., Callon M. \& Latour B. (1988). - Á quoi tient le succès des innovations ? 1: L'art de l'intéressement, gérer et comprendre. Ann. Mines, 11, 4-17.

13. Lemery B. (2003). - Les agriculteurs dans la fabrique d'une nouvelle agriculture. Sociol. Travail, 45 (1), 9-25.

14. Akrich M., Callon M. \& Latour B. (2006). - Sociologie de la traduction. Textes fondateurs (C. Méadel, édit.). Presses des Mines, Paris, 304 pp.

15. Ancey V., Ickowicz A., Corniaux C., Manoli C. \& Magnani S. (2009). - Stratégies pastorales de sécurisation chez les Peuls du Ferlo (Sénégal). J. Africanistes, 78 (1-2). Page web: http://africanistes.revues.org/2280 (consultée le 16 octobre 2014).

16. Bouyer J., Solano P., Cuisance D., Itard J., Frézil J.-L. \& Authié E. (2010). - Trypanosomosis: control methods. In Infectious and parasitic diseases of livestock (J. Bouyer, P. Solano, D. Cuisance, J. Itard, J.-L. Frézil, E. Authié \& G. Uilenberg, édit.). Éditions Lavoisier (Tec \& Doc), Paris, 1936-1943.

17. Seck M.T., Bouyer J., Sall B., Bengaly Z. \& Vreysen M.J.B. (2010). - The prevalence of African animal trypanosomoses and tsetse presence in Western Senegal. Parasite, 17 (3), 257265.

18. Ba Diao M. (2005). - Situation et conditions de développement de la production laitière intensive dans les Niayes au Sénégal. Thèse de Doctorat, Université Cheikh Anta Diop, Dakar, 138 pp.

19. Ba Diao M. (2001). - Urban-rural linkages for animal products supply in Dakar. Development Planning Unit, University College, Londres.

20. Ba Diao M. (2004). - Situation et contraintes des systèmes urbains et périurbains de production horticole et animale dans la région de Dakar. Cah. Agric., 13 (1), 39-49.

21. Dia D. (2009). - Les territoires d'élevage laitier à l'épreuve des dynamiques politiques et économiques: éléments pour une géographie du lait au Sénégal. Thèse de Doctorat, Université Cheikh Anta Diop, Dakar, 336 pp.
22. Darré J.-P. (1999). - La production de connaissance pour l'action. Éditions de la Maison des sciences de l'homme, Institut national de la recherche agronomique, Paris, 244 pp.

23. Kouamo J., Sow A., Lèye A., Sawadogo G.J. \& Ouédraogo G.A. (2009). - Amélioration des performances de production et de reproduction des bovins par l'utilisation de l'insémination artificielle en Afrique subsaharienne et au Sénégal en particulier : état des lieux et perspectives. Rev. Afr. Santé Prod. Anim., 7 (3-4), 139-147. Page web: http://eismv.org/ IMG/pdf/KOUAMO_et_al._RASPA_6_3-4_2009139-148.pdf (consultée le 25 novembre 2014).

24. Bonfiglioli A.M. (1990). - Pastoralisme, agro-pastoralisme et retour : itinéraires sahéliens. Cah. Sci. Hum., 26 (1-2), 255266.

25. Corniaux C. (2005). - Gestion technique et gestion sociale de la production laitière : les champs du possible pour une commercialisation durable du lait. Cas des modes de production actuels du Delta du fleuve Sénégal. Thèse de doctorat, Institut national agronomique de Paris-Grignon, Paris, 258 pp.

26. Vreysen M.J.B., Saleh K., Mramba F, Parker A., Feldmann U., Dyck V.A., Msangi A. \& Bouyer J. (2014). - Sterile insects to enhance agricultural development: the case of sustainable tsetse eradication on Unguja Island, Zanzibar, using an areawide integrated pest management approach. PloS Negl. Trop. Dis., 8 (5), e2857.

27. Rogers E. (1983). - Diffusion of innovations. Free Press, New York, $576 \mathrm{pp}$.

28. Stachurski F. \& Lancelot R. (2006). - Foot-bath acaricide treatment to control cattle infestation by the tick Amblyomma variegatum. Med. Vet. Entomol., 20 (4), 402-412.

29. Stachurski F. (2000). - Invasion of West African cattle by the tick Amblyomma variegatum. Med. Vet. Entomol., 14 (4), 391399. 